\title{
Perception of straightness and parallelism with minimal distance information
}

\author{
Brian Rogers $^{1,2}$ - Olga Naumenko ${ }^{2}$
}

Published online: 29 March 2016

(C) The Psychonomic Society, Inc. 2016

\begin{abstract}
The ability of human observers to judge the straightness and parallelism of extended lines has been a neglected topic of study since von Helmholtz's initial observations 150 years ago. He showed that there were significant misperceptions of the straightness of extended lines seen in the peripheral visual field. The present study focused on the perception of extended lines (spanning $90^{\circ}$ visual angle) that were directly fixated in the visual environment of a planetarium where there was only minimal information about the distance to the lines. Observers were asked to vary the curvature of 1 or more lines until they appeared to be straight and/or parallel, ignoring any perceived curvature in depth. When the horizon between the ground and the sky was visible, the results showed that observers' judgements of the straightness of a single line were significantly biased away from the veridical, great circle locations, and towards equal elevation settings. Similar biases can be seen in the jet trails of aircraft flying across the sky and in Rogers and Anstis's new moon illusion (Perception, 42(Abstract supplement) 18, 2013, 2016). The biasing effect of the horizon was much smaller when observers were asked to judge the straightness and parallelism of 2 or more extended lines. We interpret the results as showing that, in the absence of adequate distance information,
\end{abstract}

Brian Rogers

brian.rogers@psy.ox.ac.uk

Olga Naumenko

hahahihi@inbox.ru

1 Department of Experimental Psychology, University of Oxford, Oxford OX1 3UD, UK

2 Department of Psychology, Saint Petersburg State University, Saint Petersburg, Russia observers tend to perceive the projected lines as lying on an approximately equidistant, hemispherical surface and that their judgements of straightness and parallelism are based on the perceived separation of the lines superimposed on that surface.

Keywords 3-D perception - Space perception - Visual perception $\cdot$ Scene perception $\cdot$ Perspective $\cdot$ Straightness . Alignment · Parallelism

Our ability to perceive the straightness and parallelism of lines is something that we take for granted both in terms of the precision with which we can make such judgements and our prior assumptions about the computational simplicity of the task. For example, we might assume that the computational task of judging the straightness of a line is trivial given the physiological evidence of elongated receptive fields in the mammalian visual cortex (Hubel \& Wiesel, 1959, 1968). Moreover, it is not difficult to imagine that judgements of the parallelism of two lines could be achieved if there were appropriate interconnections between spatially separated, elongated receptive fields with similar orientation preferences. However, 150 years ago, von Helmholtz showed that our judgements of the straightness and parallelism of lines are not always correct, based on observations using his distorted chessboard figure (von Helmholtz, 1909; see Fig. 1). When viewed from a very close distance (corresponding to the length of line underneath the figure), the pincushion-shaped contours of the figure appear to be straight rather than curved (Oomes, Koenderink, van Doorn, \& de Ridder, 2009; Rogers \& Brecher, 2007; Rogers \& Rogers, 2009). It is important to note that these judgements are made in the peripheral visual field - the curvature of the outer chessboard contours is 


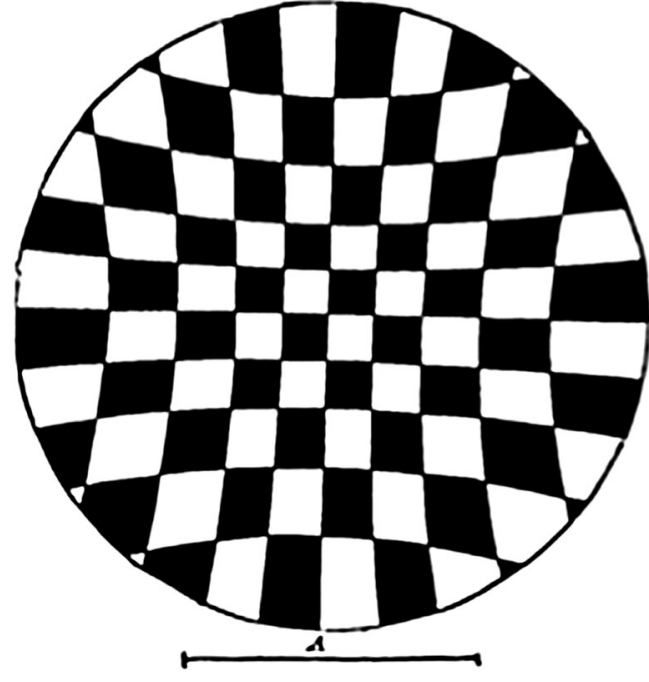

Fig. 1 When viewed from a very close distance (the length of the short horizontal line A), the curved contours of von Helmholtz's distorted chessboard figure appear to be straight. Rogers and Brecher (2007) pointed out that the images of those curved contours could also be created by equally separated contours (parallels) superimposed on a particular concave dish or dome (see Fig. 2b), and von Helmholtz (1909) confirmed that this is what observers typically perceive when viewing the figure from a close distance

readily detected if they are fixated directly, as the reader can verify for her- or himself.

It should also be noted that the perceived straightness of the contours in von Helmholtz's chessboard figure is almost certainly a consequence of inadequate distance information. ${ }^{1}$ In order to view the von Helmholtz figure on page 181 of his Treatise on Physiological Optics from a distance corresponding to the length of the line underneath, the figure has to be viewed monocularly, thereby removing nearly all the distance cues. Rogers and Rogers (2009) reported that if the same chessboard figure, subtending the same visual angle, is viewed binocularly so that there is adequate convergence and disparity information to specify the surface on which the lines are drawn, the curved contours of the figure do not appear to be straight, even when those contours stimulate the peripheral retina. This suggests that information about the shape of the surface on which the contours are drawn is crucial for making judgements about the straightness of lines. From a geometric point of view, this has to be the case because for every line on a flat 2-D surface in the world (see Fig. 2a) there

\footnotetext{
${ }^{1}$ Von Helmholtz (1909) proposed an explanation of why the curved lines of his distorted chessboard figure appear to be straight (when viewed from a close distance). That explanation was based on the idea of 'Richtkeisen' or 'direction circles'. However, as Rogers and Brecher (2007) pointed out, while that explanation might be correct for the situation of lines that are viewed in the peripheral visual field (away from the point of fixation), it is not necessarily relevant to the directly viewed lines used in the present experiments (see Rogers \& Brecher, 2007. for a detailed consideration of von Helmholtz's idea).
}

are lines on particular curved surfaces in the world that produce the same retinal image.

Rogers and Rogers (2009) also reported that observers' judgements of the parallelism of a pair of lines drawn on a concave cylindrical surface were close to veridical if there was adequate disparity information to specify the cylindrical shape of the surface on which the lines were drawn (see Fig. 2b). This means that from a single vantage point, the image created by von Helmholtz's 2-D pincushion figure could also have been produced by a set of equally spaced contours covering a particular 3-D curved surface, and this is the perceptual impression that von Helmholtz reported. At this point it is important to appreciate the difference between the geometric definition of a straight line in space - which refers to its 3-D properties - and alignment - in which three or more points are 'lined up - which is a 2-D property (Rogers \& Brecher, 2007). Three stars can appear aligned even though there is no straight line in space that could join them. Similarly, the idea of parallel $^{2}$ lines refers to the 3-D property of two lines being both straight and equally separated, whereas the idea of parallels $^{3}$ refers to the situation of two curved lines in space that are separated by a constant amount (like the lines of latitude on a globe; see Fig. 2b). As a consequence, the perceived 'straightness' and 'parallelism' of the outer lines of von Helmholtz's chessboard figure viewed from a close distance are, geometrically speaking, judgements about alignment and parallels that are seen on a concave surface.

To summarize, projective geometry shows that the perspective ambiguity ${ }^{4}$ (described by Berkeley, 1709) of lines seen from a single vantage point (and hence in a 2-D retinal image) means that it is impossible to determine (i) whether a single line is geometrically straight or aligned and curved in depth and (ii) whether a pair of lines is geometrically parallel in a single 2-D plane or nonparallel over some curved surface, whenever there is inadequate distance information. Given that the empirical evidence shows that our perceptual judgements of straightness and parallelism are influenced by the presence and availability of adequate distance information (Rogers \& Rogers, 2009), this raises the important question of how we are able to judge the straightness and parallelism of lines when distance information is weak or negligible.

In an attempt to answer this question, Rogers and Naumenko (2015) asked observers to judge the alignment ${ }^{5}$ of three

\footnotetext{
${ }^{2}$ In geometry, parallel lines are lines in a plane that do not meet.

${ }^{3}$ Equally spaced curves on a sphere are referred to as parallels of latitude, viz., the lines of latitude on a globe.

${ }^{4}$ Note that this perspective ambiguity is a consequence of using point or line stimuli. However, if one considers the world of surfaces that we inhabit, the situation is quite different since other sources of depth and distance information are available.

${ }^{5}$ We are making a judgment of alignment when, for example, we see the Pole star as being aligned with the pointers in the Great Bear. Note that there is no straight line joining the stars because the stars are at different distances.
} 


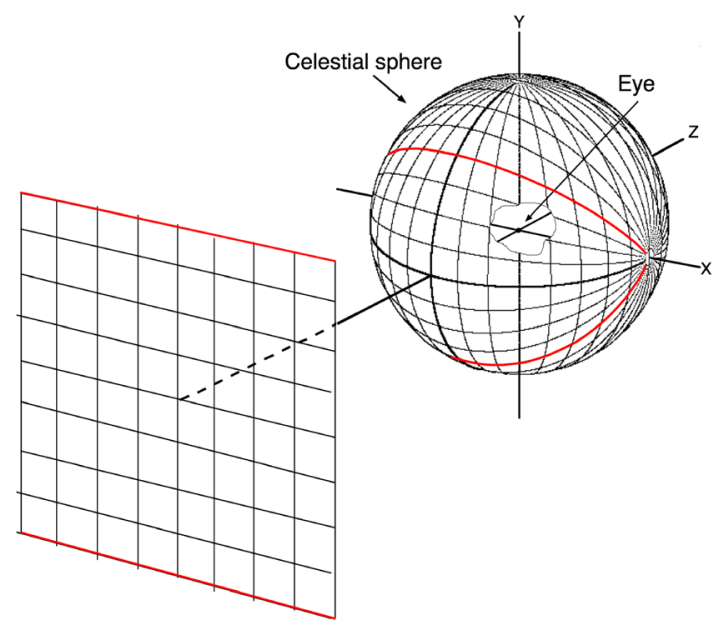

Fig. 2 (a) Any straight line in the world projects to a great circle (a line of longitude) on von Helmholtz's celestial sphere surrounding the observer's eye. To be physically aligned, the loci of the three stars would have to lie on a single great circle spanning von Helmholtz's celestial sphere. The converse, however, is not true. Given the ambiguities of inverse projection, an image coincident with a great circle does not guarantee that the line creating it is geometrically straight. (b) Lines that are parallels $^{3}$ (separated by a fixed amount) on a particular cylindrical

isolated, artificial 'stars' projected on to the dome of surface $(\sim 13 \mathrm{~m})$ meant that there was only weak information from vergence, accommodation, and binocular disparities about the distance and shape of the dome's surface. Despite this lack of information, it is important to stress that all observers perceived the array of stars projected on to the dome to be lying on an approximately hemispherical surface. This is likely to be a consequence of either the 'equidistant tendency' or the 'specific distance tendency' proposed by Gogel (1965) and is supported by the fact that the real stars in the night sky also appear to lie on a single, relatively close, hemispherical surface despite the vastly different astronomical distances of the individual stars.

In order to consider the results of Rogers and Naumenko's (2015) experiment, it is important to bear in mind that the horizon was always visible in their experiments (quite deliberately) and therefore quite likely to influence observers' judgements of the three artificial 'stars' projected onto the dome's surface. ${ }^{6}$

To be physically aligned, the loci of the three stars would have to lie on a single great circle spanning von Helmholtz's celestial sphere. ${ }^{7}$ Given that the planetarium dome is an approximate hemisphere, a straight line projected onto the planetarium dome from a position of the observer's eye would also correspond to a great circle on the celestial sphere. However, it is also important to remember that the elevations (angular separations) of points lying on any great circle increase and

\footnotetext{
${ }^{6}$ In a control experiment, in which only the three 'stars' were visible and the horizon line was not visible, observers' judgements of alignment were close to veridical.

${ }^{7}$ Visual directions in von Helmholtz's celestial sphere are specified by elevation and azimuth using spherical geometry (see Rogers \& Brecher, 2007)
}

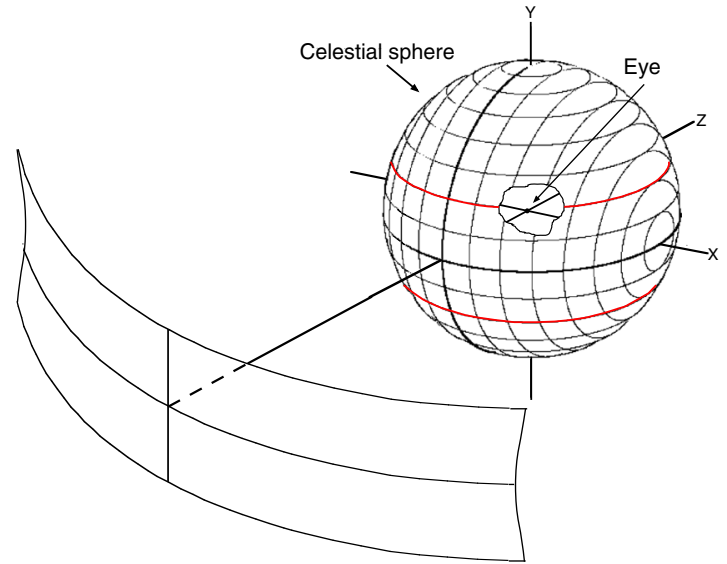

surface (equidistant) project to lines of latitude (parallels) on von Helmholtz's celestial sphere. It follows that lines that are elevated by a constant amount above the horizon on the planetarium dome project to lines of latitude on the celestial sphere. Note that because the dome of the planetarium is hemispherical and the observer is at the centre, the images projected onto the dome are equivalent to (isomorphic with) those on the celestial sphere

decrease with respect to the great circle of the horizon line (see Fig. 2a). This is equivalent to saying that the angular separation of parallel lines in the world varies along their length as a function of the distance from the observer.

Rogers and Naumenko (2015) reported that observers' judgements of alignment were, in fact, biased away from the veridical, great circle locations and towards equal elevation locations (see Fig. 3). The extent of bias increased with the angular separation of the two outer 'stars' and decreased with the separation of those stars from the horizon plane. They interpreted their results as being consistent with the perception of the three artificial 'stars' lines lying on the curved surface of the planetarium dome (for whatever reason) rather than along a geometric, straight line in space. In other words, observers' judgements were biased towards making judgements about parallels (constant angular separation) of the three 'stars' from the visible horizon, rather than strict geometric parallelism (a pair of great circles).

It was these results that prompted us to investigate the perception of straightness and parallelism in extended lines rather than the alignment of isolated stars. As with the previous experiments, the horizon line between the ground and the star-covered 'night sky' was always visible. Four different experimental situations were investigated: (1) the perception of 'straightness" (alignment) in a single line connecting two endpoints that were equally elevated above the horizon and separated by $90^{\circ}$ of horizontal azimuth; (2) the perception of 'straightness' and parallelism in a pair of lines located at two

\footnotetext{
${ }^{8}$ The single quotation marks around the word 'straightness' are used here and throughout the paper because observers were asked to adjust the line(s) until all points on the line appeared to align (lined up) and to ignore any perceived curvature in depth.
} 

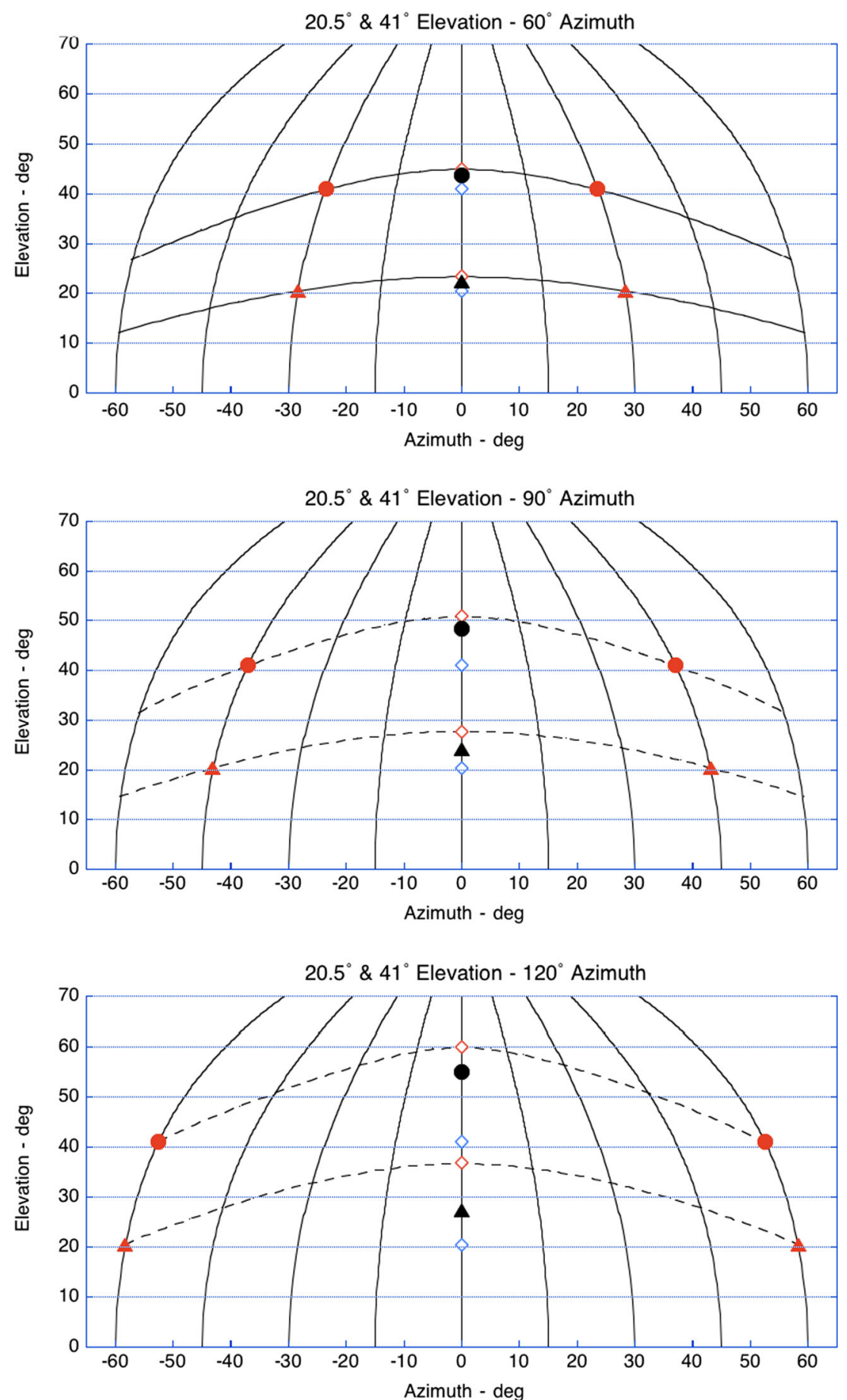

Fig. 3 Average elevation settings for the symmetrical trials in which the two 'stars' were elevated to the same extent $\left(20.5^{\circ}\right.$ or $\left.41^{\circ}\right)$. Black symbols are the data points. Solid red symbols are the locations of the outer 'stars'.
Open red diamonds and dashed lines are the locations of the veridical, great circle locations and open blue diamonds, equal elevation locations (from Rogers \& Naumenko, 2015). (Color figure online.)

The experimental hypothesis in all four experiments was that, in the absence of adequate information about distance, the perception of 'straightness' and/or parallelism of the projected lines would be biased away from the geometrically correct great circle locations and towards locations that had a constant angular separation either between the different lines 
or between the lines and the visible horizon. In addition, it was hypothesized that such biases are the consequence of seeing the lines as located on a curved surface in depth rather than lying in a single flat plane.

\section{Method}

\section{Observers}

Nine observers took part in the experiment (four male and five female), including the two authors. All observers had previous experience of taking part in psychophysical experiments.

\section{Apparatus}

A wide-angle DLP projector (BenQ MW820ST) was used to project the images of dashed lines onto the dome $(23.5 \mathrm{~m}$ in diameter) of the St. Petersburg Planetarium from a position close to the centre of the dome. The maximum size of the projected image was $90^{\circ}$ in the horizontal azimuth plane and approximately $50^{\circ}$ in vertical elevation directly in front of the observer. The projector was enclosed within a light-tight box in order to exclude any stray light from the ventilation slots.

The observer stood alongside the projector so that the lens of the projector was close to the observer's eyes. This ensured that the geometry of the observer's field of view corresponded to geometry of the projected image (see Appendix). The bottom of the projected image coincided with the horizontal plane through the projector lens. Observers used a trackball and up/ down cursor keys to modify the characteristics of the displayed images.

The locations of all points and lines projected onto the dome's surface were specified with respect to the horizontal plane through the projector lens. Elevation with respect to that plane was specified in a particular azimuth direction, and the azimuth within that plane was specified with respect to the straight ahead, that is, using longitudinal azimuth and latitudinal elevation axes (gun-turret model; Howard \& Rogers, 1995). Note that according to this system, pixels in the same vertical column of the projector's image were isomorphic with vertical lines of longitude on von Helmholtz's celestial sphere (see Fig. 2a). As a consequence, the projected images of those vertical lines converged towards a point directly above the observer's head on the hemispherical surface of the planetarium dome (just as the global lines of longitude converge towards the north pole of a world globe). Because the projector was designed to project a rectangular image on a frontal screen, pixels in a given horizontal row in the projector's image projected to great circles elevated above the horizontal plane on the dome's hemispherical surface (see Figs. 2a and Appendix).

Eight different experimental conditions were investigated: (1) Single lines (with three subconditions), (2) Pairs of parallel lines (with three subconditions), (3) Asymmetric lines, and (4) Multiple horizontal lines. Only every 10th pixel of the dashed lines of the projected images was illuminated in order to eliminate the aliasing artefacts that would be present in a continuous line. In addition to the projected lines, the planetarium dome was illuminated with an array of stars depicting the night sky and the horizon defined by a dimly visible skyline.

\section{Experiment 1: Single lines}

Stimuli In each of three different subconditions, a single 'horizontal' line was displayed at a particular elevation above the horizontal plane. In order to compare the present results with those of Rogers and Naumenko (2015) using isolated 'stars', the outer ends of the lines (at $\pm 45^{\circ}$ azimuth from the straightahead) had fixed elevations of either $14.43^{\circ}, 22.2^{\circ}$ or $30.68^{\circ}$. The curvature of lines with respect to the fixed outer ends was adjustable and covered the range from (i) a line that had a constant elevation above the horizontal plane (a line of latitude on the von Helmholtz's celestial sphere (see Fig. 2b) to (ii) a line corresponding to an elevated great circle between the east and west poles on the same celestial sphere (see Fig. 2a). ${ }^{9}$

Note that the elevation of a great circle above the horizontal plane is not constant but instead is maximal directly in front of the observer and decreases to the left and right (see Fig. 2a). For the observer, however, all great circles are "straight ${ }^{10}$ in the sense that their loci would correspond to the image created by a taut piece of string stretched between the outer ends. Whether such a line is perceived to be geometrically straight or curved in depth is an empirical matter. If the observer chose a setting in which all points on the line are aligned (i.e., a great circle on the celestial sphere), the elevation at the centre of the line would be $20^{\circ}, 30^{\circ}$, or $40^{\circ}$, respectively, in the three subconditions. On the other hand, if the observer chose a setting that corresponded to a line of constant elevation above the horizontal, the elevation of the centre of the line would be the same as that of the outer ends (i.e., $14.43^{\circ}, 22.2^{\circ}$ or $30.68^{\circ}$ ). The trackball enabled observers to make continuous adjustments of the curvature of the projected line, and they were encouraged to 'bracket' their final setting from above and below.

Procedure In all four experiments, observers were given practice trials to familiarize themselves with the effects of the different adjustments in the different conditions. No feedback was given. In the Single line trials, observers were asked to move the trackball back and forth until the line appeared to be 'straight'

\footnotetext{
${ }^{9}$ In all four experiments, the range of possible 'horizontal' lines actually extended beyond the fixed reference points of a great circle line and a line of constant elevation so that observers could not use the end points of the adjustment range to influence their settings.

10 'Straight' is used in the sense of all points on the line being aligned, ignoring any perceived curvature in depth.
} 
an adjustment procedure. They were told to ignore any change in the apparent distance to the line across the field of view (i.e., its curvature in depth) and to concentrate on setting the line so that it would appear 'straight' (aligned) "like a taut piece of string across their visual field." Observers were encouraged to look at the line and, because it was a long line, to move their eyes to view its entire extent. When the observer was satisfied that the line appeared to be 'straight', the experimenter reset the position of the trackball and the observer made a second setting of the apparent straightness of the line.

Results The two adjustment settings for each observer in a particular condition were first averaged before the means and standard deviations over the nine observers were calculated. The results are presented graphically in Fig. 4. The graphs show that the average observer settings (dashed lines) deviated away from the veridical, great circle loci and towards equal elevation loci, in a similar way to Rogers and Naumenko's (2015) results using an alignment task with three isolated 'stars'. A convenient way of expressing the magnitude of the bias away from the great circle location and towards the equal elevation location is to express the average observer setting in terms of the percentage of the difference between the veridical, great circle location, and an equal elevation location.

For the line whose outer ends were elevated by $14.43^{\circ}$ (see lowest graph, Fig. 4), the average observer setting of the curved line was $18.17^{\circ}$ at its highest point, directly in front of the observer. This shows a bias of $1.83^{\circ}$, that is, $34.8 \%$ of the way between the elevation of the veridical, great circle location $\left(20^{\circ}\right)$ and the equal elevation location $\left(14.43^{\circ}\right)$. In other words, the

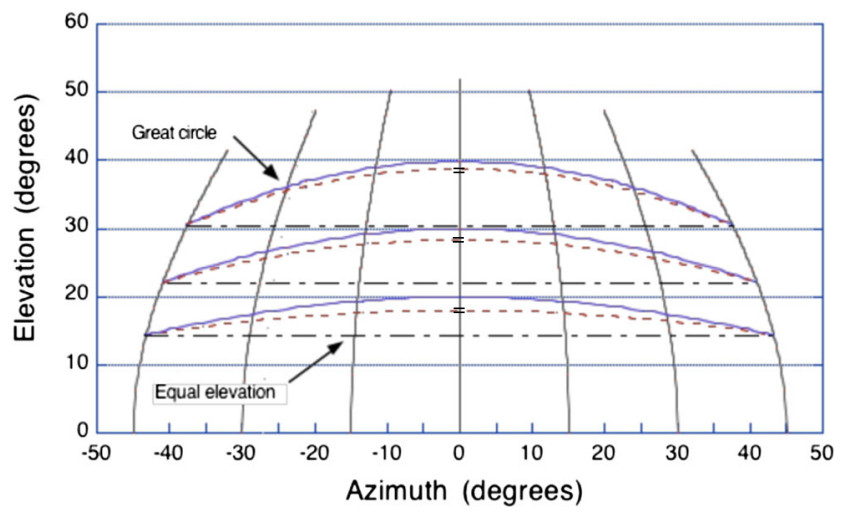

Fig. 4 Observer settings of perceived alignment of the lines as a function of the elevation of the outer ends of the lines $\left(14.43^{\circ}, 22.2^{\circ}\right.$, or $\left.30.68^{\circ}\right)$. The solid (blue) lines represent the positions of the veridical, straight line loci and the dot-dash black lines the loci of equal elevation settings. The dashed (red) lines represent the average of the observer settings. The error bars at the centres of the lines show \pm 1 standard error of the mean of the nine observers' average settings. (Color figure online.) bias was just over one third of the difference between the two reference lines, as can be seen in Fig. 4 . For the line whose outer ends were elevated by $22.2^{\circ}$ (middle graph), the average observer setting was $28.43^{\circ}$, that is, a smaller bias of $20.1 \%$ and for the line whose outer ends were elevated by $30.68^{\circ}$ (uppermost graph), the average observer setting was $38.94^{\circ}$ (i.e., an even smaller bias of $11.4 \%$ ). Note that the variability over the observers' settings is very small in both this and the subsequent experiments. The standard deviations of the observers' settings were $0.39^{\circ}, 0.39^{\circ}$, and $0.27^{\circ}$ for the three conditions, and the standard errors a factor of three $(\sqrt{ } 9)$ smaller. Single-sample $t$ tests for each of the three elevation conditions revealed that the observed differences from the great circle locations were all significant, $p<.01$. The decreasing effect of the horizon line on the observers' settings with an increase in elevation of the line is consistent with the findings of Rogers and Naumenko (2015) using isolated 'stars'. Note, however, that the magnitude of the bias for continuous lines observed in the present experiment was somewhat less (for similar elevations of the outer end of the lines) than that found previously in the alignment of three isolated 'stars' (see Fig. 3).

\section{Experiment 2: Pairs of lines}

Stimuli On these trials, a pair of lines was projected onto the planetarium dome. Unlike the Single line trials, where the elevation of the outer ends was kept constant and the observer's adjustments of curvature affected the elevation of the central portion of the line, in these trials the elevations of the each of the pair of lines directly in front of the observer were fixed and the observer's adjustments of curvature affected the elevation of the outer portions of the two lines. Three different subconditions were investigated in which the elevations of the centres of the pairs of lines were set to either $20^{\circ}$ and $30^{\circ}, 30^{\circ}$ and $40^{\circ}$, or $20^{\circ}$ and $40^{\circ}$. As with the single line trials, observers used the trackball to adjust the curvature of the lower line over the range from (i) a line that had constant elevation above the horizontal plane to (ii) a great circle between the east and west poles on the same celestial sphere. Observers were subsequently asked to use the up/down cursors keys to adjust the relative curvature of the upper line with respect to the lower line.

Procedure On these trials, observers were again asked to move the trackball back and forth until the lower of the two lines appeared to be 'straight' using the same criteria as for the Single line trials. Once set, observers were asked to use the up/down cursor keys to adjust the curvature of the upper of the two lines until the two lines appeared to be 'parallel' (i.e., equally separated). Observers were then encouraged to readjust the trackball 
position and the cursor keys until both lines appeared to be 'straight' (aligned) and 'parallel'. Once again, observers were encouraged to look directly at the lines and to move their eyes to view the entire extent of the two lines. After resetting the positions of the trackball and cursor keys, the observer made a second setting of the apparent straightness and parallelism of the two lines.

Results The two adjustment settings for each observer were first averaged before the means and standard deviations over the nine observers in each condition were calculated. Once again, there was an impressive consistency in the observers' settings with standard deviations of less than $0.5^{\circ}$ in all cases. The results are presented graphically in Fig. 5. As with the Single line results, the average 'straight line' settings of observers (dashed lines) in all three conditions were biased away from the great circle loci (solid lines) in the direction of equal elevation settings. This was true of both the upper and lower lines and in all three elevation conditions. The existence of a bias suggests that the presence of the visible horizon line influenced observers' judgements. Note, however, that the magnitudes of the biases were small. In the case of the $20^{\circ}$ and $30^{\circ}$ lines, the average observer settings of the outer ends of the lines were $15.52^{\circ}$ and $23.62^{\circ}$ compared with great circle values of $14.43^{\circ}$ and $22.21^{\circ}$, respectively. When expressed as a percentage of the difference between the veridical, great circle locations and the equal elevation locations, the biases were $19.6 \%$ and $18.28 \%$ (for the $20^{\circ}$ and $30^{\circ}$ lines, respectively). Effects of a similar magnitude were also found for the $30^{\circ}$ and $40^{\circ}$ condition and the $20^{\circ}$ and $40^{\circ}$ condition. In spite of the relatively small effect sizes, single-sample $t$ tests revealed that the differences between each of the average settings and the corresponding great circle locations were all statistically significant, $p<.01$. The smaller biases shown in all three Pairs of lines conditions (and the closeness of the settings to the veridical, great circle loci) suggest that the presence of the visible horizon line had much less of an influence on observers' judgements of straightness and parallelism when two lines were visible rather than one.

\section{Experiment 3: Asymmetric lines}

Stimuli On these trials, a pair of lines was projected onto the planetarium dome, but instead of the lines being symmetric in their elevation with respect to the straight ahead, the elevations of the outer ends of the lines were asymmetrically elevated by $20^{\circ}$ and $40^{\circ}-$
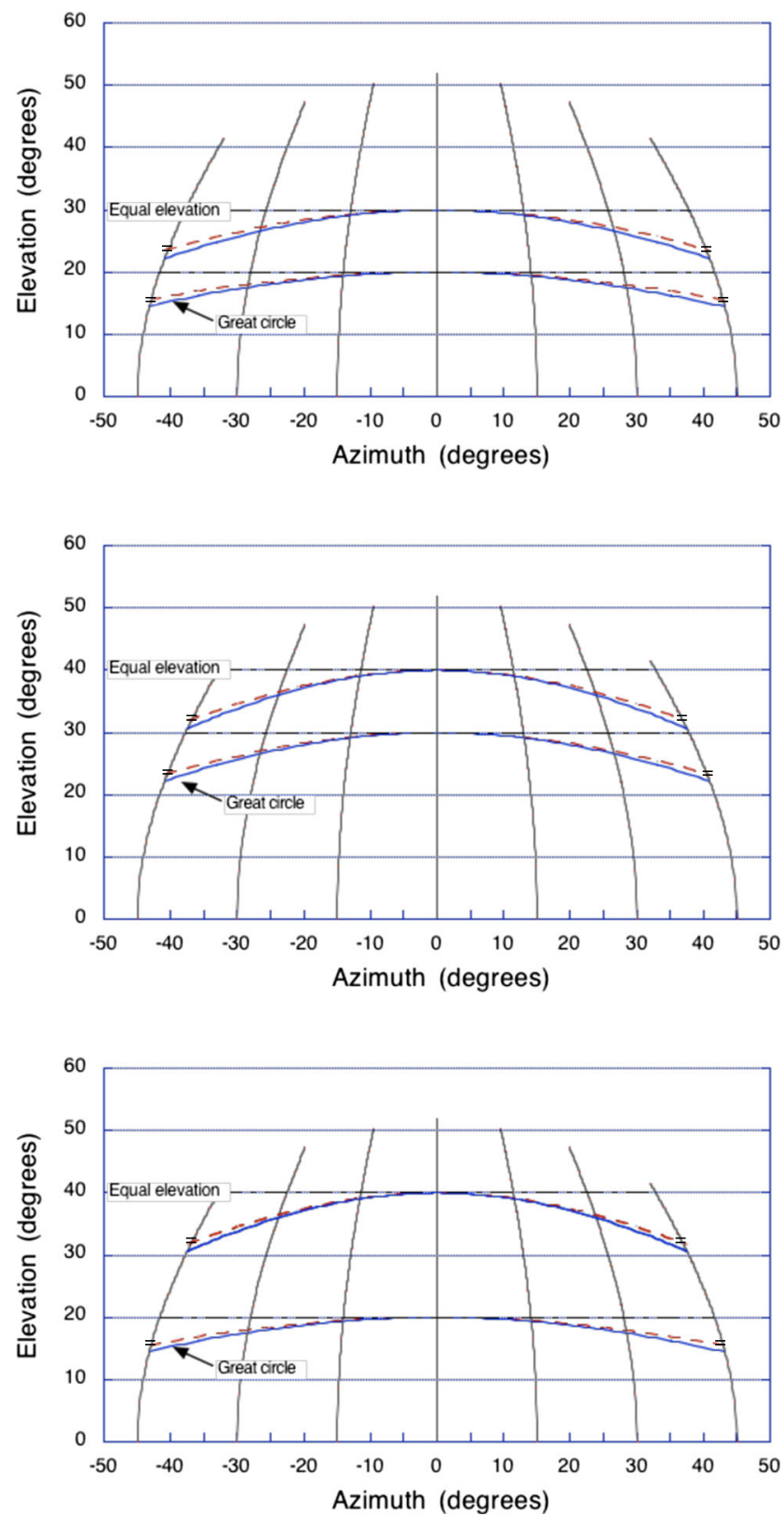

Fig. 5 Observer settings of perceived 'straightness' and 'parallelism' of the two projected lines for the three different conditions $\left(20^{\circ}\right.$ and $30^{\circ}, 30^{\circ}$ and $40^{\circ}$, or $20^{\circ}$ and $40^{\circ}$ ). The solid (blue) lines represent the positions of the veridical, straight line loci of the two lines, and the horizontal dot-dash black lines the loci of equal elevation settings. The dashed (red) lines are the average of the observer settings. The error bars show \pm 1 standard error of the mean of the nine observers' average settings of 'straight' (aligned) and 'parallel'. (Color figure online.)

one line being inclined from $20^{\circ}$ to $40^{\circ}$ from left to right and the other from $40^{\circ}$ to $20^{\circ}$ from left to right over their horizontal azimuth separation of $90^{\circ}$. Once again, observers were required to adjust the curvature of the lines over a range from (i) lines that were great circles on the celestial sphere (i.e., geometrically 'straight' from the observer's viewpoint) to (ii) lines 
that increased linearly in elevation as a function of azimuth. ${ }^{11}$

Procedure On the Asymmetric lines trials, observers used the trackball to adjust the curvatures of the two lines simultaneously. The two asymmetric lines formed a cross-like pattern between their fixed endpoints. Observers were asked to adjust the curvature of the lines until both lines appeared as 'straight' (aligned) as possible, ignoring any curvature in depth. Note that the intersection point of the two lines moved up and down as the observer made his or her adjustments. After resetting the position of the trackball, the observer made a second setting of the apparent 'straightness' of the two lines.

Results The two adjustment settings for each observer were first averaged before the means and standard deviations over the nine observers were calculated. The results are presented graphically in Fig. 6. As with the Single line results, the average 'straight line' settings of observers (dashed lines) were biased away from the great circle loci (solid line) in the direction of a linear increase in elevation locus. However, like the results for the Pairs of lines conditions, the effect was small. The elevation of the average observer setting at the centre point of the line (straight in front of the observer) was $39.5^{\circ}$ $\left(S E= \pm 0.17^{\circ}\right)$ compared with the great circle location of $40.39^{\circ}$ and a linearly increasing elevation of $30^{\circ}$. This corresponds to less than $10 \%$ of the difference between the veridical, great circle locations and the linear increase in elevation loci. Although small, a single-sample $t$ test revealed that the differences between the average setting and the corresponding great circle location was statistically significant, $p<.01$. The smaller size of the bias found in this condition (and the closeness of the settings to the veridical, great circle loci) suggests that the presence of the visible horizon line had much less of an influence on observers' judgements of straightness when the two asymmetric lines were visible.

\section{Experiment 4: Multiple horizontal lines}

Stimuli The stimuli used in these trials can be thought of as an extension of the Pairs of lines conditions (Experiment 2). The elevations of the centres of the multiple lines directly in front of the observer were fixed and set to $15^{\circ}, 20^{\circ}, 25^{\circ}, 30^{\circ}, 35^{\circ}$,

\footnotetext{
${ }^{11}$ In this experiment, the range of possible 'inclined' lines actually extended beyond the fixed reference points of a great circle line and a line of linearly increasing elevation so that observers could not use the end points of the adjustment range to influence their settings. The choice of lines that 'increased linearly in elevation' was made because we believed this to be analogous to lines that had constant elevation used in Experiments 1 and 2 . In practice, observers reported that they had no difficulty in finding an adjustment point when the two asymmetric lines appeared 'straight' somewhere between great circle lines and lines of linearly increasing elevation.
}

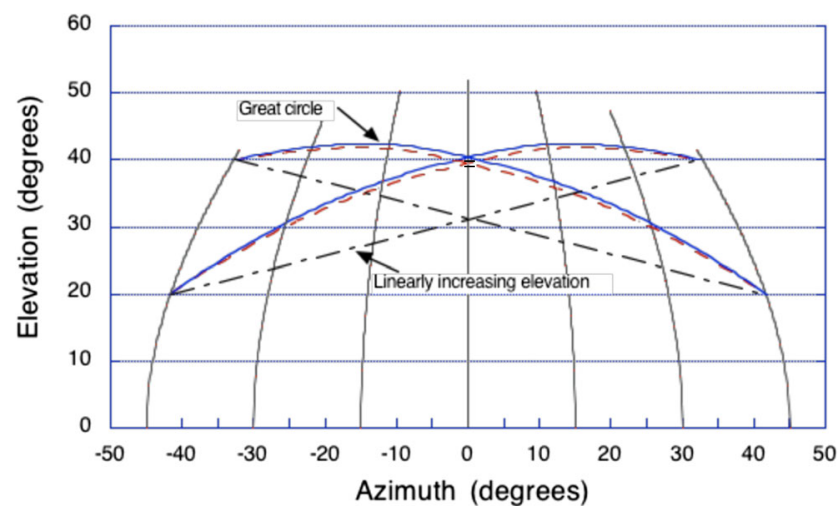

Fig. 6 Observer settings of perceived 'straightness' of the two asymmetric lines (endpoints elevated by $20^{\circ}$ and $40^{\circ}$ ). The solid (blue) lines represent the positions of the veridical, straight line loci of the two lines and the dashed (red) line, the average of the observer settings. The dot-dash black lines represent the loci of linearly-increasing elevations. The error bar at the centre shows the \pm 1 standard error of the mean of the nine observers' average settings. (Color figure online.)

$40^{\circ}$, and $45^{\circ}$. The observer's adjustments of curvature affected only the outer portions of the lines. As with the Pairs of lines trials, observers used the trackball to adjust the curvature of the lowest line over a range from (i) a line that had constant elevation above the horizontal plane to (ii) a great circle between the east and west poles on the same celestial sphere. Observers were subsequently asked to use the up/down keys to adjust the relative curvatures of the remaining lines with respect to the lower line.

Procedure In these trials, observers were again asked to move the trackball back and forth until they judged the lowest of the seven lines to be 'straight' using the same criteria as for the Single line trials. Once set, observers used the up/down cursor keys to adjust the curvature of the upper lines until they also appeared to be 'straight' and parallel to (i.e., equally separated from) each other. The change of curvature of each of the upper lines for a given up/down key adjustment was in proportion to the separation of the line from lowest line. As in the Pairs of lines trials, observers were encouraged to repeat the trackball and cursor key adjustments until all seven lines appeared to be both 'straight' and 'parallel'. As in previous experiments, observers were told to ignore any perceived change in the apparent distance to the lines across the field of view (i.e., their curvature in depth) and instead to concentrate on finding a setting where the lines appeared to be 'straight' (aligned) and 'parallel' (equally separated). After resetting the positions of the trackball and cursor keys, the observer made a second setting of the apparent straightness and parallelism of the multiple lines.

Results The two adjustment settings for each observer were first averaged before the means and standard 
deviations over the nine observers were calculated. The results are presented graphically in Fig. 7. As in all previous conditions, observers did not choose settings corresponding to the veridical, great circles (solid lines) but instead they chose settings (dashed lines) that were biased towards equal elevation loci. The magnitudes of the biases, although statistically significant $(p<.01)$ using single-sample $t$ tests, were only small (between $1^{\circ}$ and $2^{\circ}$ ) and amounted to less than $10 \%$ of the difference between the veridical, great circle locations and the equal elevation locations. The fact that the biases from the veridical, great circle loci were so small suggests that the presence of the visible horizon line had much less of an influence on observers' judgements of straightness when multiple lines were presented rather than a single line.

\section{Summary and discussion}

The purpose of the four experiments described in this paper was to investigate how good we are at judging the alignment ('straightness') and parallelism of extended lines in the absence of adequate distance information. Consider, first, a single line, viewed in an otherwise dark environment. If fixated directly, there is no reason to assume that observers would be other than veridical in a judgement of the line's straightness. On the other hand, if its image fell in the peripheral visual field, von Helmholtz's observations using his pincushion chessboard figure (see Fig. 1) might lead us to expect that our judgements would be biased away from veridicality, that is, a straight line in the periphery might be seen as curved concave with respect to the fovea (barrel distortion), and a convex curve with respect to the fovea (pincushion distortion, as in von Helmholtz's distorted chessboard pattern) might be seen as straight, but only in situations where there is inadequate information about the distance to the line (shape of the surface on which it is seen; Rogers \& Brecher, 2007; Rogers \& Rogers, 2009).

We know, however, that our perception of straightness can be biased even when extended lines are viewed directly. The Flemish physicist Minnaert (1940) noticed that a searchlight beam directed across the sky, although physically straight, appeared to be curved "highest of all in the middle and sloping down to the ground on both sides" (p. 151). Rogers and Naumenko (2015) pointed out that a similar distortion of apparent curvature can be seen in the straight-line jet trails of aircraft that are seen crossing the sky. This might seem surprising, given that both the jet trail and the horizon lines are actually "straight" ${ }^{12}$ and parallel, and the two lines would project to great circles on von Helmholtz's celestial sphere (see

\footnotetext{
$\overline{12}$ By holding up a taut piece of string, the reader can verify for her- or himself that jet trails are typically 'straight' (aligned) across the sky.
}

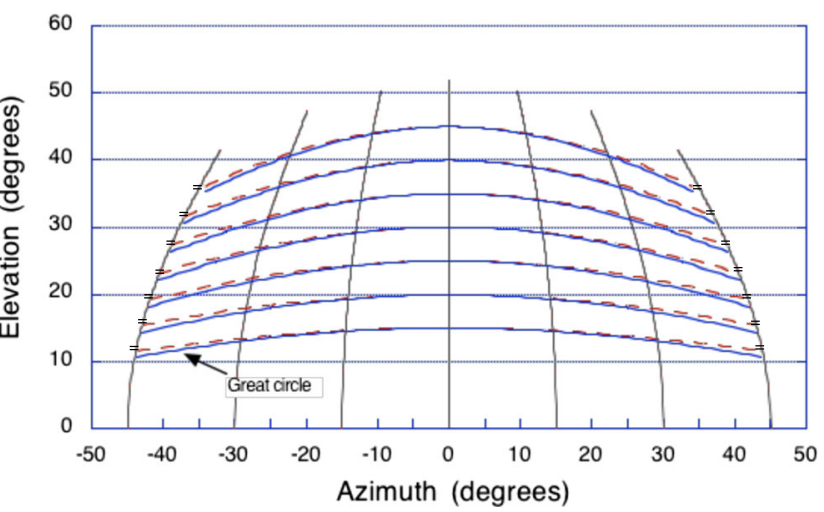

Fig. 7 Observer settings of perceived 'straightness' and parallelism of the multiple horizontal lines (centre points elevated by $15^{\circ}, 20^{\circ}, 25^{\circ}, 30^{\circ}$, $35^{\circ}, 40^{\circ}$, and $45^{\circ}$ ). The solid (blue) lines represent the positions of the veridical, great circle locations of the multiple lines and the dashed (red) lines represent the average of the observer settings. The error bars show the \pm 1 standard error of the mean of the nine observers' average settings. (Color figure online.)

Fig. 2a). The clue as to why a jet trail might be perceived as curved - "highest of all in the middle" - comes from the fact that it is seen to cross the hemispherical dome of the sky. At its start, the jet trail is typically inclined upwards from the horizon, then it becomes parallel to the horizon, before becoming inclined downwards towards the horizon. Lines that diverge and then converge are not just changing in terms of their angular separation from the horizon in the optic array or retinal image, but, more importantly, they are changing in terms of their perceived separation on a curved surface. As a consequence, such a pair of lines would not be regarded as parallel but rather as diverging and converging with respect to each other. In other words, it is not that we are 'seeing' our retinal images in which there is a changing angular separation between the two lines but rather that we see a change in the perceived separation of the lines as they traverse a particular curved surface.

The same explanation can also account for the finding that observers' judgements of the perceived alignment of the three artificial 'stars' projected onto the surface of the planetarium dome ${ }^{13}$ were biased away from the veridical, straight line loci and towards equal elevation loci (Rogers \& Naumenko, 2015). This explanation also accounts for the finding that the bias is diminished with increasing elevation of the stars from the horizon. We suggest that the same explanation can be used to account for the data found in Experiment 1 (see Fig. 4). If a single line projected onto the planetarium dome is geometrically straight (and projects to a great circle on von Helmholtz's celestial sphere) but is seen to lie on (or close to) the dome's hemispherical surface, its perceived separation from the horizon will not be constant. We suggest that the judgement of whether two lines are

\footnotetext{
${ }^{13}$ Note that the dome is perceived to be hemispherical in shape.
} 


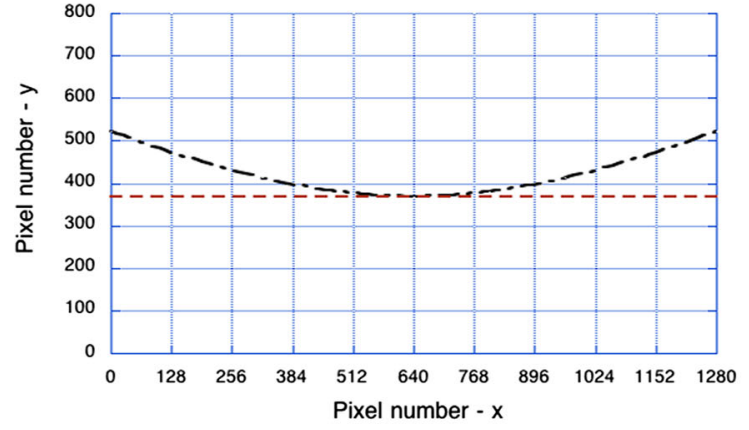

Fig. 8 The rectangular $(1,280 \times 800$ pixel $)$ display in the projector (a) created an image on the hemispherical dome of the planetarium and in the observer's optic array (b). A horizontal row of pixels in the projector's image (dashed red line) - a straight line - maps to a great circle in the optic array with its changing elevation. An upwardly curved row of pixels in the projector's image (dot-dash black line) maps to a line in the optic

'parallel' ${ }^{14}$ is based on whether those lines are perceived to have the same physical separation, and this depends on the shape of the surface on which those lines are seen. Lines that have the same physical separation on a hemispherical surface with the eye at its centre are the lines of latitude (parallels) with an equal angular separation such as the lines of latitude on the earth's surface.

Our explanation also predicts that the effect of the horizon should decrease with the increasing elevation of the line, as we have found (see Fig. 4). The fact that our results show only a bias towards those equal elevation locations (rather than a complete shift) could be due to a number of factors, including a failure to see the projected line as lying on the surface of the dome or seeing the dome as a flattened hemisphere (Kaufman \& Rock, 1962).

In Experiment 2, where two 'horizontal' lines were projected onto the planetarium dome, the results also showed a similar bias towards equal elevation settings, although the magnitude of the bias was smaller than in Experiment 1 (see Fig. 5). We suggest that this was because observers were basing their judgments on the perception of the two elevated lines so that the presence of the horizon line had less of an influence. In addition, a closer examination of the graphs in Fig. 5 shows that, although the observers' settings of straightness and parallelism in the two lines were close to their great circle locations, the angular separation between the great circles at the centres of the lines $\left(10^{\circ}\right.$ in the case of the $20^{\circ} \& 30^{\circ}$ and the $30^{\circ} \& 40^{\circ}$ lines) is not very different to the angular separation between the two lines at their endpoints $\left(7.8^{\circ}\right)$. Hence it is not surprising that observers saw even the pair of great circle lines as remaining approximately parallel (i.e., equally separated) across the planetarium dome.

The results in Experiment 3, using two asymmetric lines (see Fig. 6), also showed that observers' settings of

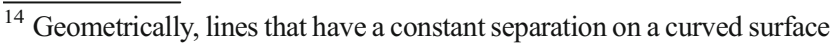
are referred to as 'parallels'.
}

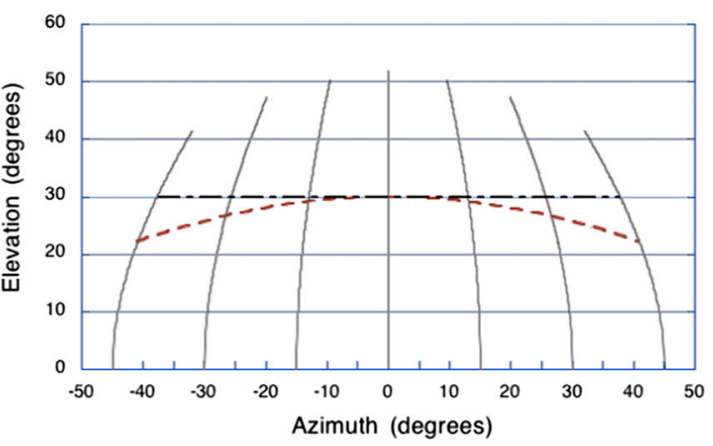

array with constant elevation. Note that the angular separations between vertical lines (lines at right angles to the horizon) in the projected image (b) do not stay constant but decrease with increasing elevation in a similar way to the separations between lines of longitude on a terrestrial globe. (Color figure online.)

straightness were close to the veridical, straight line locations. This finding is consistent with the results of Rogers and Naumenko (2015) using asymmetrically elevated isolated 'stars'. Once again, this would suggest that the presence of the visible horizon had only a very small effect on observers' judgements of the straightness of the asymmetric two lines. This is what one might expect because, while it does not sound unreasonable to suggest that observers could be influenced by the changing angular separation of a single symmetrical line from the horizon, it sounds less plausible that observers' settings could be biased by an imaginary reference line that is increasing linearly in its separation from the horizon as it crossed the dome's surface.

The images projected onto the planetarium dome in Experiment 4 comprised a set of seven 'horizontal' lines. Once again, the average observer settings (dashed lines) were biased away from the veridical great circle locations and towards equalelevation loci (see Fig. 7), but the extent of that bias was small. Such a result would be expected if observers were basing their judgments on their perception of just the elevated lines so that the presence of the horizon line had less of an influence on their judgements. In addition, Fig. 7 reveals that, although the observers' settings of straightness and parallelism in the multiple horizontal lines were close to their great circle locations, the difference between the angular separation of the great circles at the centres of the lines ( $5^{\circ}$ in all cases) was not very different to the difference in the angular separation of their endpoints. Hence, it is not surprising that observers saw even the great circle multiple lines as remaining approximately parallel (i.e., equally separated) across the planetarium dome.

\section{Conclusions}

The aim of the experiments described here was to investigate observers' abilities to judge the straightness and parallelism of extended lines in a situation where there was weak or inadequate 
distance information. The starting hypothesis was that those judgements would be influenced by the presence of the visible horizon. The reported results show that judgements of the perceived straightness of single line were significantly biased by the horizon line in a similar way to the biases reported by Rogers and Naumenko (2015) for the perceived alignment of three artificial stars. While it is reasonable to conclude that the presence of the horizon line was the cause of these biases, they reveal that judgments of straightness and parallelism by the human visual system are likely to be based on the perceived separation between two lines lying on the particular surface on which they are seen - in this case, the perceived curved surface of the planetarium dome. This is not surprising. Railway tracks receding into the distance are perceived to be (approximately) parallel because they are seen as lying on a surface that is inclined with respect to the line of sight. In other words, judgments of size (physical separation) are based on relative size with respect to features on an inclined surface. The novel feature of the present experiments is that there was little or no direct information about the distance to the lines projected onto the planetarium dome. The perceived hemispherical shape of the planetarium dome that provides the basis for making these judgements is most likely to be the result of the same 'equidistance tendency' or 'specific distance tendency' (Gogel, 1965) that is responsible for our perception of the night sky of stars as a flattened dome (Kaufman \& Rock, 1962).The principal finding from Experiment 1 - that a straight line projected onto the curved surface of a planetarium dome is perceived to be curved - is clearly related to the observation that the straight-line jet trails created by aircraft flying across the sky are also seen to be curved (Rogers \& Naumenko, 2015). This misperception is also related to the New Moon illusion, in which observers appear to be unable to perceive the imaginary straight line joining the sun and the moon as a great circle, because of the constantly changing angular separation between the line and the horizon (Rogers \& Anstis, 2013, 2016).

The results also reveal that judgements of the perceived parallelism of a pair of 'horizontal' lines, and multiple 'horizontal' lines were also affected by the presence of the visible horizon, although to a much smaller extent. One possible reason for why the biases in the average observer settings were small (and closer to the veridical, great circle locations) in these conditions is that the projected lines may not have been seen as lying on the dome's hemispherical surface but rather on some compromise surface, with a curvature somewhere between that of the dome and of a frontal surface. This possibility needs to be investigated further.

Author note The authors acknowledge the receipt of a post-doctoral research grant 8.50.2098.2013 from Saint Petersburg State University as well as grant $\mathrm{N}^{\mathrm{o}} 14-06-0030 \mathrm{~A}$ from the RFBR. We are also very grateful to all the staff of Saint Petersburg Planetarium and especially to its director, Michael Belov, for giving us the opportunity to carry out the experiment.

\section{Appendix}

The BenQ projector was designed (and independently calibrated in our experiment) so that the $1,280 \times 800$ pixel image displayed on a frontal screen would be rectangular in shape. On a frontal screen, a vertical column of pixels would remain vertical in the projected image, and a horizontal row of pixels would remain horizontal in the projected image. Note, however, that the characteristics of the projected image in the observer's optic array (and at the retina) remain the same whether the image is projected onto a frontal screen (for which it was designed) or on a hemispherical surface like the planetarium dome, as long as the observer's eye is close to the lens of the projector. Hence, a straight, vertical row of pixels in the projector's image (see Fig. 8a) projected to a great circle or (vertical) line of longitude on von Helmholtz's celestial sphere (see Fig. 8b) and a horizontal row of pixels in the projector's image projects to great circle or (horizontal) line of longitude on von Helmholtz's celestial sphere.

\section{References}

Berkeley, G. (1709). A new theory of vision. London, England: Dent and Sons.

Gogel, W. C. (1965). Equidistance tendency and its consequences. Psychological Bulletin, 64, 153-163.

Howard, I. P., \& Rogers, B. J. (1995). Binocular vision and stereopsis. New York, NY: Oxford University Press.

Hubel, D. H., \& Wiesel, T. N. (1959). Receptive fields and functional architecture of monkey striate cortex. Journal of Physiology, 195, 215-243.

Hubel, D. H., \& Wiesel, T. N. (1968). Receptive fields of single neurones in the cat's striate cortex. Journal of Physiology, 148, 574-591.

Kaufman, L., \& Rock, I. (1962). The moon illusion I. Science, 136, 953 961.

Minnaert, M. (1940). Light and colour in the open air. London, England: G. Bell and Sons.

Oomes, A. H. J., Koenderink, J. J., van Doorn, A. J., \& de Ridder, H. (2009). What are the uncurved lines in our visual field? A fresh look at Helmholtz's chessboard. Perception, 38, 1284-1294.

Rogers, B. J., \& Anstis, S. M. (2013). The new moon illusion. Perception, 42(Abstract supplement), 18.

Rogers, B. J., \& Anstis, S. M. (2016). The new moon illusion. In A. Shapiro \& D. Todorovic (Eds.), The Oxford compendium of visual illusions. New York, NY: Oxford University Press (in press).

Rogers, B. J., \& Brecher, K. (2007). Straight lines, 'uncurved lines', and Helmholtz's 'great circles on the celestial sphere'. Perception, 36, $1275-1289$.

Rogers, B. J., \& Naumenko, O. (2015). The new moon illusion and the role of perspective in the perception of straight and parallel lines. Attention, Perception and Psychophysics, 77(1), 249-257.

Rogers, B. J., \& Rogers, C. (2009). Visual globes, celestial spheres, and the perception of straight and parallel lines. Perception, 38, 1295-1312.

von Helmholtz, H. (1909). Helmholtz's treatise on physiological optics. New York, NY: Dover 1962. (J. P. C. Southall, Trans., from the third German edition of Handbuch der Physiologischen Optik. Hamburg, Germany: Voss). 